\title{
Study on the effect of jackfruit pancake implementation in combating malnutrition among the tribes of Bargarh district
}

\author{
Shweta Parida AND LiPSa Dash
}

\begin{abstract}
Nutritional status of children is a proxy indicator for assessing the entire population health status and one of the major predicators of child survival. India is considered as the motherland of jackfruit.Pancakes is another popular wheat-based product. The aim of this study was to plot the nutritional deficiency in the tribal community, prepare a Nutritional food supplements by conducting nutritional and sensory evaluation for the acceptance of the product and to compare the initial health status with the post implemented supplied pancake. The study was carried out in Lahanda villages under Attabira Block of Bargarh district with a sample size 45 (6-58 months). In laboratory condition pancake was prepared from jack fruit seed paste combined with rice paste in a proportion of $0,15,30$ and $45 \%$, respectively. Nutritional and its sensory evaluation has been done keeping in mind for the nutritional enhancement. The study revealed that after three months of counseling the variation in weight was increased to $26.44 \%$ Green and decreased $2.4 \%, 22 \%$ in yellow and red, respectively according to the WHO growth Chart.
\end{abstract}

Key Words : Health status, Nutritional food, Tribal, Jackfruit, Pancake, Sensory evaluation

How to cite this article : Parida, Shweta and Dash, Lipsa (2017). Study on the effect of jackfruit pancake implementation in combating malnutrition among the tribes of Bargarh district. Food Sci. Res. J., 8(2): 343-348, DOI : 10.15740/HAS/FSRJ/8.2/343348. 